\title{
Source-reconstruction of the sensorimotor network from resting-state macaque electrocorticography
}

\author{
Hindriks $^{1}$, R., Micheli ${ }^{2,3}$, C., Bosman ${ }^{4}$, C.A.,

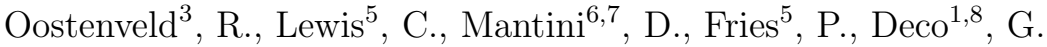

June 1, 2018

1. Center for Brain and Cognition, Computational Neuroscience Group, Department of Information and Communication Technologies, Universitat Pompeu Fabra (UPF).

2. Institut des Sciences Cognitives Marc Jeannerod, UMR 5304 CNRS, Bron, France.

3. Donders Institute for Brain, Cognition and Behaviour, Radboud University Nijmegen, 6525 EN Nijmegen, The Netherlands.

4. Cognitive and System Neuroscience Group, Swammerdam Institute for Life Sciences, Center for Neuroscience, University of Amsterdam, 1098 XH, Amsterdam, The Netherlands.

5. Ernst Strüngmann Institute (ESI) for Neuroscience in Cooperation with Max Planck Society, 60528 Frankfurt, Germany.

6. Research Center for Motor Control and Neuroplasticity, KU Leuven, Tervuursevest 101, 3001 Leuven, Belgium.

7. Functional Neuroimaging Laboratory, IRCCS San Camillo Hospital, via Alberoni 70, 30126 Venice Lido, Italy.

8. Instituci Catalana de la Recerca i Estudis Avanats (ICREA), Universitat Pompeu Fabra. 


\begin{abstract}
The discovery of hemodynamic (BOLD-fMRI) resting-state networks (RSNs) has brought about a fundamental shift in our thinking about the role of intrinsic brain activity. The electrophysiological underpinnings of RSNs remain largely elusive and it has been shown only recently that electric cortical rhythms are organized into the same RSNs as hemodynamic signals. Most electrophysiological studies into RSNs use magnetoencephalography (MEG) or scalp electroencephalography (EEG), which limits the spatial resolution with which electrophysiological RSNs can be observed. Due to their close proximity to the cortical surface, electrocorticographic (ECoG) recordings can potentially provide a more detailed picture of the functional organization of resting-state cortical rhythms, albeit at the expense of spatial coverage. In this study we propose using source-space spatial independent component analysis (spatial ICA) for identifying generators of resting-state cortical rhythms as recorded with ECoG and for reconstructing their functional connectivity. Network structure is assessed by two kinds of connectivity measures: instantaneous correlations between band-limited amplitude envelopes and oscillatory phase-locking. By simulating rhythmic cortical generators, we find that the reconstruction of oscillatory phase-locking is more challenging than that of amplitude correlations, particularly for low signal-to-noise levels. Specifically, phase-lags can both be over- and underestimated, which troubles the interpretation of lag-based connectivity measures. We illustrate the methodology on somatosensory beta rhythms recorded from a macaque monkey using ECoG. The methodology decomposes the resting-state sensorimotor network into three cortical generators, distributed across primary somatosensory and primary and higher-order motor areas. The generators display significant and reproducible amplitude correlations and phase-locking values with non-zero lags. Our findings illustrate the level of spatial detail attainable with source-projected ECoG and motivates wider use of the methodology for studying resting-state as well as event-related cortical dynamics in macaque and human.
\end{abstract}




\section{Introduction}

Blood-oxygen level-dependent functional MRI (BOLD-fMRI) has demonstrated that in the absence of structured stimuli and explicit cognitive tasks i.e. during the resting-state, hemodynamic fluctuations in cortical gray-matter of humans and macaques are correlated across regions, forming resting-state networks (RSNs) [24, 11, 18, 42]. The electrophysiological correlates of hemodynamic RSNs have only recently been discovered using scalp electroencephalography (EEG) and magnetoencephalography (MEG) Although the high temporal resolution of scalp EEG and MEG allow for investigating the temporal dynamics of RSNs, their spatial resolution is limited, due to the large distance of $[44,12,4,33,40]$.the sensors to the cortex and the spatial lowpass filtering effect of the skull (in case of EEG) $[26,47]$. In electrocorticography (ECoG), electric potentials are recorded directly from the cortical surface and therefore have higher spatial resolution than EEG and MEG, albeit at the expense of spatial coverage. This allows studying resting-state cortical activity with high temporal and spatial resolution $[29,25]$.

ECoG measurements are often analyzed on the sensor-level as it as commonly believed that sourcereconstruction does not improve spatial resolution, because the electrodes are close to the current generators $[63,2]$. Human and macaque cortices, however, are convoluted with sulci that can be up to three centimeters deep (in human) and this complicates the interpretation of sensor-level ECoG recordings. Even in the absence of such convolutions, ECoG recordings are contaminated by volumeconduction. Theoretical and experimental studies have demonstrated that even intra-cortical local field potentials (LFPs) suffer from volume-conduction too $[38,31]$ and that their spatial resolution can be improved by applying source-reconstruction techniques [53, 16, 32]. These findings motivate the development of volume-conductor models and source-reconstruction methods for ECoG recordings $[21,66,8,39,14,55,9]$. Currently, however, source-reconstruction of ECoG recordings is relatively underdeveloped, as compared with scalp EEG and MEG recordings and existing studies have largely focused on epileptiform activity $[21,66,8,39,14,55,9]$.

In this study, we investigate the feasibility of reconstructing source-level RSNs from sensor-level ECoG recordings in macaque monkey. Due to the limited spatial coverage of the electrode grid and good coverage of the central sulcus and frontal cortex, in both simulation and application, we restrict analysis to sensorimotor regions. From BOLD-fMRI studies in macaque monkeys, it is known that these regions are involved in network formation [35]. The proposed analysis pipeline is based on the assumption that the ECoG potentials are generated by a finite number of spatially-extended current generators and comprises the following three steps: 1. Source-projection of the sensor-level ECoG data, 2. Generator delineation using spatial ICA, and 3. Reconstruction of functional connectivity between the generators. The main difference between our method and the commonly used methods in EEG/MEG resting-state studies is that, instead of reconstructing the functional connectivity between all pair's of source-space locations $[33,48]$, the functional connectivity analysis is confined to the identified generators. Sourceprojection was done with the minimum-norm estimate with a prior in the spherical Fourier domain [52]. This prior reduces surface bias in the inverse reconstructions and is especially suited for localizing spatially-extended generators [52]. Generator identification was done with independent component analysis (ICA) [43, 1, 37, 40]. Functional connectivity was measured using amplitude envelope correlations and complex-valued oscillatory phase-locking. Although amplitude correlations are often used in resting-state scalp EEG and MEG studies [48, 40], amplitude signals fluctuate on timescales that are at least one order of magnitude larger than the periods of the oscillations. For example, in the case of beta oscillations with a peak-frequency of $16 \mathrm{~Hz}$, the oscillation periods center around $62.5 \mathrm{~ms}$, while amplitudes fluctuate on time-scales in the order of seconds. Thus, although amplitude correlations allow characterizing the spectral content of RSNs by considering band-limited amplitude fluctuations $[44,12,4,33,40]$, they do not allow to investigate latencies between oscillatory signals, which have been hypothesized to play a fundamental role in selective routing of information $[19,20]$. 
To assess the feasibility of reconstructing source-space RSNs from sensor-level ECoG data, we simulated networks of spatially-extended oscillatory generators, projected their activity to the ECoG sensor-grid, and subsequently applied the reconstructing pipeline. Due to the limited spatial coverage of the electrode grid and its good coverage of the central sulcus and frontal cortex, in both simulations and application to empirical data, we focused on the sensorimotor network. Our simulations demonstrate that cortical generators can be recovered from ECoG sensor data, and that their amplitude correlations can be accurately reconstructed, even for noisy data. Leakage correction by symmetric orthogonalization, which is frequency used to infer resting-state functional connectivity from MEG data $[33,48]$, was found to underestimate the amplitude correlations. Furthermore, oscillatory phase-locking values could also be recovered, although this required a higher signal-to-noise ratio. For noisy data, we found that the relative phases between local oscillations can both be over- and under-estimated. In particular, as a consequence of higher-order volume-conduction effects [13], local oscillations that were instantaneously coupled (i.e. with zero phase-difference) can seem lagged with respect to each other. These effects complicate the interpretation of interaction indices that only take into account first-order volume-conduction effects [46, 61] and motivate the development and use of more refined indices [13].

We illustrate the methodology using two ECoG recording sessions of a single macaque monkey, displaying strong sensorimotor beta $(\approx 16 \mathrm{~Hz})$ oscillations, which have been implicated in several cognitive functions $[20,3]$. We identified three generators; two in primary motor cortex of which one extending into the frontal cortex, and one in primary somatosensory cortex, which display significant and reproducible amplitude correlations and imaginary phase-locking values, in line with intra-cortical LFP recordings $[58,5]$.

\section{Materials and Methods}

\subsection{Recordings and pre-processing}

Electrocorticographic (ECoG) data were recorded on two different resting-state sessions from a single macaque monkey using a 252-electrode subdural grid covering a large part of the left-hemispheric cortical surface [57]. During the recording sessions, which we will refer to as session 1 and session 2 , the monkey was situated in front of a wide screen in an otherwise dark room. Simultaneously recorded electro-ocular signals were manually inspected for the presence of eye-blinks and saccades, which confirmed that the monkey was awake with eyes open throughout both sessions. The duration of session 1 and 2 was 11.5 and 13 minutes, respectively. Because a substantial number of electrodes overlying posterior parts of the cortex were noisy and did not show oscillatory activity, the analysis was confined to the 122 most anterior electrodes. Remaining noisy electrodes (two and one in session 1 and 2, respectively) were replaced by averages of the neighboring electrodes. The signals were filtered using a notch filter at $50 \mathrm{~Hz}$, a fourth-order zero-phase Butterworth lowpass filter with cut-off frequency $60 \mathrm{~Hz}$, and subsequently down-sampled from $1000 \mathrm{~Hz}$ to $500 \mathrm{~Hz}$ and converted to averagereference montage. Five consecutive minutes of artifact-free data were selected from each session. Figure 1A shows the electrode-averaged power spectra of the selected data and Figure 1B shows tensecond time-series from the 20 most anterior electrodes. In both sessions, strong beta oscillations with a peak-frequency of about $16 \mathrm{~Hz}$ could be observed. To extract the beta oscillations, the data were filtered between 12 and $20 \mathrm{~Hz}$ with a zero-phase 4-th order Butterworth bandpass filter.

\subsection{Construction of electrocorticographic leadfields}

In order to construct a monkey-specific ECoG leadfield matrix, the ECoG electrodes were localized relative to the monkey's brain by the following procedure. During implantation surgery, pictures of the monkey brain were taken with a Nikon D5000 camera (42882848-pixel resolution, RGB color space) to document the position of the metal contacts relative to anatomical landmarks (sulci, gyri, vasa). The pictures, together with a triangulation of the cortical surface, were used as inputs to a custom-made 
interactive MatLab program to mark the electrode contacts. The triangulation of the cortical surface was obtained by segmenting a pre-surgical T1-weighted MRI (magnetization prepared rapid-gradient echo MPRAGE volumes acquired with Siemens tfl3d $1_{\text {ns }}$ pulse sequence with flip angle $=8^{\circ}$; FOV $=$ $18 \mathrm{~cm}$, voxel size $=0.7 \times 0.7 \times 0.7 \mathrm{~mm}^{3}$, number of slices $=192$, head first supine) into cerebrospinal fluid, white and grey matter using monkey-specific tissue priors [45] in SPM8. The manually obtained electrode coordinates were corrected to achieve nominal inter-electrodes distance $(2.5$ or $3 \mathrm{~mm}$ according to the grid finger) and to follow geodesic curvilinear trajectories.

The source-space for the leadfield matrix was obtained from the T1-weighted MRI image using Caret software [64], which yielded a high-resolution triangulation of the monkey's mid-thickness cortical surface comprising 21339 vertices. The source-space was defined by placing current dipoles at each of the vertices and the dipole orientations were treated as free parameters that were estimated from the data. Subsequently, a two-layer boundary-element method (BEM) realistic volume-conductor model (dura and white/gray matter boundary) was constructed using OpenMEEG [22] and FieldTrip [50]. The BEM model yields the electric potential at the vertices of the outermost surface, generated by unit-strength current dipoles located at the vertices of the cortical mesh, which is enclosed by both layers [49]. OpenMEEG requires four inputs: triangulated boundary surfaces of the head conductors with spherical topology, the position of the current dipoles, the electrode positions, and the conductivity values within the two compartments.

The outer boundary surface (i.e. the inner skull) was constructed by applying morphological operations (opening, closing, smoothing) and thresholding the segmented T1-weighted MRI volume, followed by triangulation [17]. Spherical topology was ensured by correcting the triangulation using custom geometry functions in FieldTrip. For numerical reasons related to calculation speed and intrinsic software limitations, we distributed no more than $10^{4}$ vertices on this surface. Vertex locations were chosen adaptively, with a denser coverage over the electrode grid. The inner boundary surface (i.e. epicortical surface) was obtained by an inward shift of the outer boundary surface by $1 \mathrm{~mm}$. The conductivity values of the outer and inner compartments were set to 1 and $0.33 \mathrm{~S} / \mathrm{m}$, respectively [41]. The obtained ECoG leadfield matrix was subsequently converted to yield average-montage electric potentials at the 122 selected electrodes.

\subsection{Source-space projection}

The data were projected to the source-space using Harmony [52], which is a smoothed version of the classical minimum-norm estimate (MNE) designed to reduce surface-bias. Let $m$ and $n$ denote the number of electrodes and source-space locations (i.e. cortical vertices), respectively, and let $t$ denote the number of samples. The $3 n \times t$-dimensional source-activity matrix $J$ is obtained by applying the Harmony inverse operator $G_{\lambda}^{\sharp}$ to the $m \times t$-dimensional data matrix $X$ :

$$
J=G_{\lambda}^{\sharp} X,
$$

where

$$
G_{\lambda}^{\sharp}=\Sigma\left(G \Sigma G^{T}+\lambda I_{m}\right)^{-1},
$$

where $\lambda \geq 0$ is the noise regularization parameter, the $3 n \times 3 n$ matrix $\Sigma$ denotes the a priori covariance matrix of $J, I_{m}$ denotes the $m \times m$ identity matrix, and $T$ denotes matrix transpose. Harmony models $\Sigma$ as

$$
\Sigma=H \Sigma_{h} H^{T},
$$

where $H$ denotes the $3 n \times 6\left(l_{\max }+1\right)^{2}$ Harmonic transformation matrix, which contains the spherical harmonics up to and including Harmonic degree $l_{\max }=20$. The $6\left(l_{\max }+1\right)^{2}$-dimensional matrix $\Sigma_{h}$ 
denotes the a priori source covariance matrix expressed in the Harmonic basis and is modeled as a diagonal matrix with diagonal entries equal to $1 /\left(1+l^{p}\right)$, where $p=0.5$ controls how fast the $a$ priori source variance decreases with degree and therefore controls the level of spatial smoothing in the source reconstructions [52]. The spherical harmonics were calculated on the vertices of a triangulated spherical mesh that corresponds to the triangulation of the fiducial cortical surface [64]. After sourcespace projection, the vector-valued time-series at each cortical vertex was reduced to a scalar-valued time-series by projecting it onto its first eigenvector.

An appropriate value for $\lambda$ was determined by applying the $L$-tangent norm criterion [6] to 100 randomly chosen samples and averaging the obtained values. The $L$-tangent norm criterion selects the value of $\lambda$ that minimizes the speed along the (logarithm of the) $L$-curve. Let

$$
L(\lambda)=(\|J\|,\|X-G J\|)
$$

be the $L$-curve [27], then $\lambda$ is chosen as

$$
\lambda^{*}=\operatorname{argmin} \frac{d}{d \lambda} \log _{10}(L(\lambda)),
$$

which was obtained by calculating the $L$-curve on the (logarithm of the) interval $[-6,0]$ in steps of 0.1 and by approximating $d / d \lambda$ in these points by (forward) finite differences.

\subsection{Reconstruction of functional networks}

After obtaining the scalar time-series at every cortical vertex, the generators i.e. network nodes are identified using spatial independent component analysis (ICA). Spatial ICA is applicable in this context, because the number of band-limited amplitude samples is an order of magnitude less than the number of cortical vertices. Due to the linearity of the source-projection, however, the effective spatial dimension of the band-limited amplitudes is limited by the number of electrodes and hence could be reduced considerably by projecting it to a low-dimensional subspace (using PCA, for example), so that temporal ICA can be used as well. We found, however, that in our simulations this approach was unable to delineate the true generators and we therefore used spatial ICA. The components were extracted using the FastICA package for Matlab, which implements Hyvarinen's fixed-point algorithm. The non-linearity $g$ used in the fixed-point algorithm was $g(u)=u^{3}$, and the algorithm was initialized with a random guess. Maximum number of iterations was set to 1000 and the stopping criterium was set to $\epsilon=0.0001$.

The appropriate number of generators $k$ is determined by inspection of the eigenspectrum of the sensor-level data matrix. Before applying ICA, the rank of data matrix was reduced by retaining only the first $k$ eigenvectors. This was done because in our simulations this improved the delineation of generators. Because in practice it is not always clear how to set $k$, we carried out simulations in which $k$ was set to a different value than the true number of generators. Spatial ICA is used only to estimate the number of generators and to reconstruct their spatial profiles because their associated time-courses generally not well resemble the true generator time-series. After delineation of the generators, the generator time-courses were obtained by selecting the time-course of the cortical vertex that correlated most strongly with the generators' time-course. Studies on source-level resting-state networks using MEG and scalp EEG typically focus on either amplitude correlations [48] or oscillatory phase-locking and coherence [30]. We therefore study the reconstruction of both types of functional connectivity: amplitude correlations and oscillatory phase-locking.

Let $s_{1}(t)$ and $s_{2}(t)$ be the reconstructed time-courses of two identified generators. The amplitude correlation $\rho$ between $s_{1}(t)$ and $s_{2}(t)$ is defined as the Pearson correlation between the instantaneous amplitudes $a_{1}(t)$ and $a_{2}(t)$ of $s_{1}(t)$ and $s_{2}(t)$, which are defined as

$$
a_{1}(t)=\sqrt{s_{1}^{2}(t)+\tilde{s}_{1}^{2}(t)},
$$


where $\tilde{s}_{1}(t)$ is the Hilbert transformation of $s_{1}(t)$ and similarly for $s_{2}(t)$. The amplitude correlation ranges between -1 and 1 and measures the strength of the instantaneous linear dependence between $a_{1}$ and $a_{2}$. It is known that for MEG and scalp EEG data, amplitude correlations are corrupted by volume-conduction effects and several studies have proposed schemes to correct for this [33, 10, 48, 65]. To assess if such a correction is useful for source-level network reconstruction from ECoG data, we also computed the amplitude correlations between orthogonalized reconstructed time-courses [48]. This method consists in removing zero-lag correlations between the generator's time-courses, prior to calculating amplitude correlations, and can be implemented through a pre-whitening transformation applied to the matrix of generator time-courses.

Oscillatory phase-locking between reconstructed time-courses $s_{1}(t)$ and $s_{2}(t)$ was measured by the complex-valued phase-locking factor $\nu$, which is defined as

$$
\nu=\left\langle\exp i\left(\phi_{1}(t)-\phi_{2}(t)\right)\right\rangle
$$

where $\phi_{1}(t)$ and $\phi_{2}(t)$ denote the instantaneous phases of $s_{1}(t)$ and $s_{2}(t), i$ denotes the imaginary unit, and the brackets denote temporal averaging [51]. The phase-locking factor $\nu$ is complex-valued; its magnitude ranges between 0 and 1 and measures the strength of oscillatory phase-locking, while its phase provides information about the time-delay between $s_{1}(t)$ and $s_{2}(t)$.

\subsection{Construction of null-data}

To test for significant amplitude-correlation and phase-locking magnitude between a pair of delineated generators, we used phase-randomized surrogate data [59]. We shortly describe their construction. Let $x=\left(x_{1}, \cdots, x_{n}\right)$ and $y=\left(y_{1}, \cdots, y_{n}\right)$ be the reconstructed time-courses of two generators. To construct a surrogate copy of the pair $(x, y), x$ and $y$ are first transformed to the Fourier domain, yielding $X=\left(X_{1}, \cdots, X_{n}\right)$ and $Y=\left(Y_{1}, \cdots, Y_{n}\right)$. Subsequently, the entries of $X$ and $Y$ are multiplied by random phases, yielding $\tilde{X}$ and $\tilde{Y}$, where

$$
\tilde{X}_{n}=X_{n} e^{i \phi_{n}}
$$

and

$$
\tilde{Y}_{n}=Y_{n} e^{i \psi_{n}},
$$

where $\phi=\left(\phi_{1}, \cdots, \phi_{n}\right)$ and $\psi=\left(\psi_{1}, \cdots, \psi_{n}\right)$ are conjugate-symmetric random vectors whose entries are uniformly distributed in the interval $[0,2 \pi]$. A surrogate copy $(\tilde{x}, \tilde{y})$ of $(x, y)$ is obtained by applying the inverse Fourier transform to $\tilde{X}$ and $\tilde{Y}$. The conjugate symmetry of $\phi$ and $\psi$ ensures that the surrogate time-series $\tilde{x}$ and $\tilde{y}$ are real-valued. The surrogate time-series $\tilde{x}$ and $\tilde{y}$ have the same autocorrelation functions as $x$ and $y$, respectively, but lack cross-correlations [59] and can hence be used to approximate the null-distributions of amplitude-correlations and phase-locking magnitudes. Specifically, if $m$ surrogate copies of $(x, y)$ are generated and if the observed value of the amplitudecorrelation or phase-locking magnitude exceeds all $m$ surrogate values, the observed value is significant with $p$-value $<1 /(m+1)$.

To test for significance of lagged oscillatory phase-locking, as measured by the imaginary part of the phase-locking factor, the above surrogate data is not appropriate, because the randomization not only destroys the imaginary part of the phase-locking factor, but the entire complex-valued phaselocking factor. To test the null-hypothesis of zero imaginary phase-locking, only the imaginary part should be destroyed, while keeping the real part. Appropriate surrogate data can be constructed by taking the real part of the Fourier transforms of $x$ and $y$ and by subsequently multiplying by the same random phase-vector to both Fourier transforms. Thus, the randomized Fourier transforms, denoted by $\hat{X}_{n}$ and $\hat{Y}_{n}$, are given by

$$
\hat{X}_{n}=\operatorname{Re}\left[X_{n}\right] e^{i \phi_{n}}
$$


and

$$
\hat{Y}_{n}=\operatorname{Re}\left[Y_{n}\right] e^{i \phi_{n}},
$$

respectively, where Re denotes taking the real part, and where $\phi=\left(\phi_{1}, \cdots, \phi_{n}\right)$ and is conjugatesymmetric random vector whose entries are uniformly distributed in the interval $[0,2 \pi]$. Note that in the definition of $\hat{X}_{n}$ and $\hat{Y}_{n}$ the same phase-vector $\phi$ is used. Similar as above, a surrogate copy $(\hat{x}, \hat{y})$ of $(x, y)$ is obtained by applying the inverse Fourier transform to $\hat{X}$ and $\hat{Y}$. The effect of using the same phase-vector for both $\hat{X}_{n}$ and $\hat{Y}_{n}$ is that, in addition to preserving the auto-correlation functions of $x$ and $y$, the randomization preserves the Fourier phase-differences between $x$ and $y$ and hence their phase-locking value [54]. The effect of taking the real part of the Fourier transforms is that $(\hat{x}, \hat{y})$ approximately have the same cross-covariance function as $(x, y)$, with the difference that it has been symmetrized. In other words, the surrogate time-series only retain instantaneous phase-locking and are therefore appropriate for testing the null-hypothesis of no lagged interactions. $p$-values are obtained in the same way as above.

\subsection{Simulation of functional networks}

The reconstruction pipeline was tested by simulating resting-state oscillations comprising a network of $k$ spatially-extended cortical generators and projecting the activity to the ECoG electrode grid. The center location of the $i$-th generator is denoted by $x_{i}$ and its amplitude at location $x$ is modeled as

$$
a_{i}(x)=\bar{a}_{i} \exp \left(-d\left(x, x_{i}\right) / \lambda_{i}\right),
$$

where $\bar{a}_{i}$ denotes the generators' (maximal) amplitude, $\lambda_{i}$ its characteristic scale, and $d\left(x, x_{i}\right)$ the geodesic distance between $x$ and $x_{i}$ along the cortical surface. The time-course of the $i$-th generator was simulated by generating a Gaussian white-noise signal, bandpass filtering it $\pm 2 \mathrm{~Hz}$ around the generators' peak frequency $f_{i}$, and subsequently normalizing it to variance one. Because the time-courses are generated independently for each generator, they are non-phase-locked oscillatory time-series $\bar{s}_{i}$ with uncorrelated amplitude envelopes. Correlations are incorporated by mixing the generators' timecourses according to

$$
s_{i}(t)=\bar{s}_{i}(t)+\epsilon \sum_{j \neq i} \bar{s}(t)_{j}
$$

where $\epsilon \geq 0$ is the mixing coefficient that controls the overall level of phase-locking and amplitudecorrelation, and where $j$ runs over all generators except the $i$-th. The mixed time-courses were subsequently normalized to variance one. This yields a network of oscillatory generators which are phaselocked at zero lag and whose amplitudes are instantaneously correlated. Lagged phase-locking was incorporated by translating the time-course of generator $i$ over $\tau_{i}$ milliseconds, relative to the first generator. Simulation of this network yields an $n \times t$-dimensional matrix $S$, where $n$ is the number of cortical vertices and $t$ the number of samples, containing the simulated time-courses in its rows.

The simulated data were subsequently projected to the ECoG electrode-grid and measurement noise was added, yielding a simulated data matrix

$$
X=G_{n} S+\sigma \xi
$$

where $G_{n}$ denotes the scalar leadfield matrix, which is derived from the vector leadfield matrix by taking the current dipoles to be perpendicular to the cortical surface, and $\xi$ denotes Gaussian noise with covariance matrix taken from the empirical data, filtered between 60 and $80 \mathrm{~Hz}$, and scaled to have maximal value one. Since this frequency band still contains physiological signal, $\xi$ models a combination of spontaneous cortical background activity and sensor noise. The noise intensity $\sigma$ was chosen to obtain a signal-to-noise ratio (SNR) of $\eta$ (a free parameter), and in its calculation, the signal power was taken to be the average power across electrodes. 
We placed $k=5$ generators at locations (partially) underneath the electrode grid and will refer to these as generators 1, 2, 3, 4, and 5. Their spatial profiles are shown in Figure 2A. Generator 1 is located on the posterior wall of the central sulcus, generators 2 and 5 in the supplementary motor area, just outside the grid, generator 3 on the wall of the superior arcuate sulcus, and generator 4 on the crown of the anterior central gyrus. To make the network realistic and challenge the reconstruction method, the generators' amplitudes, characteristic scales, peak-frequencies, and time-lags differed between generators and are listed in Table 1. Observation time and sampling frequency were set equal to those of the experimental data ( 5 minutes and $500 \mathrm{~Hz}$, respectively). For each choice of the parameters, we averaged the results over ten realizations. Figure $2 \mathrm{~B}$ shows a ten-second epoch of the simulated ECoG data from the 20 most anterior electrodes.

Table 1: Simulation parameters. Listed are the parameters, their symbols, and values/ranges. The subscript $i$ refers to the $i$-th generator. Time-lag refers to the time-lag relative to the first generator.

\begin{tabular}{lcc}
\hline \hline Parameter & Symbol & Values/range \\
\hline Number of generators & $k$ & 5 \\
Maximal amplitude & $\bar{a}_{i}$ & $2,3,1,4,2$ \\
Characteristic scale (mm) & $\lambda_{i}$ & $4,3,5,2,3$ \\
Peak-frequency (Hz) & $f_{i}$ & $16,15,17,18,16.5$ \\
Mixing coefficient & $\epsilon$ & 0.5 \\
Time-lag (ms) & $\tau_{i}$ & $10,20,30,0$ \\
Signal-to-noise ratio (dB) & $\eta$ & $10,20,30$
\end{tabular}




\section{Results}

\subsection{Identification of cortical generators}

In resting-state MEG studies, functional connectivity is usually reconstructed by computing a connectivity index between all pairs of source locations (cortical vertices in this case) [30, 48]. In contrast, the central idea of our method is to first identify the generators of resting-state cortical activity and subsequently to restrict functional connectivity analysis to the time-courses of the identified generators. In this section, we assess the accuracy of reconstructing the generator's spatial profiles.

To set an appropriate value for the number of generators $k$, we inspected the eigenspectra of the simulated ECoG data and they show that $k=5$ is an appropriate choice for the number of generators for all three noise-levels. For comparison with the true generators, the reconstructed generators were ordered to match the true generators, based on their spatial correlations. For all three noise-levels, the identified generators could be unambiguously paired with the true generators. Figure 3A shows the spatial correlations between the true and reconstructed generators for the three noise-levels. The correlations are generally high and roughly equal for all noise-levels, except for generator 3, which is less well reconstructed in case of noisy data $(10 \mathrm{~dB})$. To understand why this is the case, we have displayed the amplitude profile of generator 3 and that of its reconstructions for all three noise-levels (Figure 3B). The figure shows that although generator 3 is accurately reconstructed for low and mid noise levels (20 and $30 \mathrm{~dB}$ ), for noisy data it gets mixed with nearby generator 5 as well as with projected sensor-noise (designated by arrows). The reason why this happens with generator 3 instead of one of the other generators most likely is that generator 3 has the lowest amplitude (Table 1).

These simulations demonstrate that spatial ICA is able to identify cortical generators from restingstate band-limited amplitude fluctuations, at least for data that is not too noisy. We note that if the focus is on functional connectivity, rather than on the spatial profiles of the generators, these results are still relevant, because inaccurately reconstructed profiles do impact the quality of the functional connectivity reconstruction, as the latter is based on the former. This will be discussed in detail in Sections 3.2 and 3.3. When dealing with empirical data, it is generally impossible to asses the accuracy of the reconstructed generator profiles, since it depends on a combination of several factors such as the spatiotemporal characteristics of the sensor-noise, the accuracy of the forward model, and the organization of cortical activity, most of which are unknown. The simulations above, however, suggest an ad hoc way of assessing the accuracy of the reconstructions, which is to inspect the spatial profiles of the reconstructed generators and to see if they overlap and/or consist of multiple cortical regions (as in the case above).

In empirical data, it is generally unknown how many generators are active, and it is therefore instructive to consider the effect of setting $k$ to a higher or lower value than the true number of generators. We repeated the simulations above while setting $k=6$ and $k=4$. Figure 3C shows the spatial correlations between the five true generators and the five unambiguously paired reconstructed generators in the case of $k=6$. The figure shows that the correlations are roughly equal to those obtained by setting $k=5$. Thus, setting $k$ too high does not distort the spatial profiles of the true generators. Surprisingly, the spatial profile of generator 3 is more accurately reconstructed than when setting $k=5$, especially for noisy data (compare Figure 3A and C). To see why this is the case, consider Figure 3D, which shows the spatial profile of the spurious generator 6 for the $10 \mathrm{~dB}$ case. The figure shows that instead of mixing generator 5 and projected sensor-noise into reconstructed generator 3 , generator 5 and the projected sensor-noise now form reconstructed generator 6 . The reason why generator 3 is more accurately reconstructed when setting $k$ to high, is thus that all contaminating factors (generator 5 and projected sensor-noise) are not mixed in and instead, form an additional generator. When the number of generators is set too low $(k=4)$, the weakest generator (generator 3 ) is missed, but the

other generators are correctly identified and their spatial correlations with the true generators remain 
high (Figure 3E).

These simulations suggest that there is an advantage in setting $k$ higher than the true number of generators as this tends to unmix the true generators by grouping generator copies and projected sensor-noise into additional generators. We not that this advantage is not unique to (spatial) ICA as it can be observed with other decomposition techniques such as clustering and non-negative matrix factorization. The disadvantage is that in practice, the true number of generators is generally unknown, and it might therefore not be possible to decide which generators are spurious. An ad hoc criterion that happens to work in our simulations is to visually inspect the spatial profiles of the reconstructed generators. Spurious generators are then set apart by their composite nature and/or overlap with other reconstructed generators. We stress that there is no guarantee for the general validity of this criterion. From a practical perspective, setting the number of generators too low is less disadvantageous because although generators might be missed, the spatial profiles of the identified generators remain accurate. The subset of identified generators can subsequently be used for reconstructing a subnetwork of the true functional network.

\subsection{Reconstruction of band-limited amplitude correlations}

After identifying the generators, their functional connectivity can be reconstructed. In this section we assess the feasibility of reconstructing the generators' band-limited amplitude correlations and of the effect of leakage correction.

The top row of Figure 4A shows the reconstructed amplitude correlations between all pairs of generators for all three noise-levels in case the correct number of generators is chosen (i.e. $k=5$ ) and the middle row shows the reconstruction errors, that is, the differences between the true and reconstructed amplitude correlations. Positive and negative entries correspond to positive and negative bias, respectively. For mid and low noise-levels $(20$ and $30 \mathrm{~dB})$ the amplitude correlations are accurately reconstructed, while for noisy data $(10 \mathrm{~dB})$, the amplitude correlations of generator 3 with the other generators, particularly generator 1 and 5, are biased. Specifically, the amplitude correlation of generator 3 with generator 5 and 1 is over- and underestimated, respectively. To understand what is causing this, we note that because the generator's time-courses are extracted based on their reconstructed spatial profiles, the quality of the reconstructed amplitude correlations depends on the quality of the reconstructed profiles. Since for noisy data, generator 5 gets mixed into generator 5 (see Section 3.1 ), the amplitude correlation between generator 3 and 5 is overestimated. Mixing of generator 5 into generator 3 is also responsible for the bias with the other generators. For example, the amplitude correlation between generator 3 and 1 is underestimated, because the true amplitude correlation between generator 1 and 5 is weaker than that between generator 1 and 3 . These simulations thus show that inaccurate reconstruction of a generators' spatial profiles, or more specifically, the location of maximum amplitude, can lead to bias in the generators' amplitude correlations with other generators.

In the previous section, we have shown that if the number of generators is set too high $(k=6)$, the true generators can still be identified, and that for noisy data $(10 \mathrm{~dB})$, the reconstructed spatial profiles are in fact more accurate, as compared to setting $k$ to the true number of generators $(k=5)$. As a consequence, the reconstructed amplitude correlations also become more accurate, because the amplitude envelopes used for their reconstruction are based on the spatial profiles of the reconstructed generators. This is shown in Figure 4B. In particular, for noisy data, the connectivity of generator 3 with generators 1 and 5 is now accurately reconstructed. In practice, however, this is of limited use because the true number of generators is unknown and there is no systematic way of deciding which reconstructed generator is spurious and should be discarded from the analysis. An ad hoc criterion that happens to work in our simulations, however, is to discard generators that are too strongly correlated with one or more of the other generators. Because the spurious generator is formed by combining generator 5 and projected sensor-noise (see Section 3.1), its time-course is strongly correlated with that of 
generator 5 (Pearson correlation of 0.70). Combined with its unusual spatial profile, this generator can be classified as spurious. Similarly as for spatial profiles, there is no guarantee for the general validity of this criterion. In the previous section we have also shown that when the number of generators is set too low $(k=4)$, the reconstructed spatial profiles are still accurate (see Figure $3 \mathrm{E}$ ), although the weakest generator (generator 3) is missed. Consequently, the amplitude correlations are accurately reconstructed as well (see Figure 4C).

To assess the effect of leakage correction on the reconstructed amplitude correlations, we repeated the simulations, with the difference that amplitude correlations are calculated between symmetrically orthogonalized generator time-courses [48]. The bottom row of Figure 4A, B, and C show the resulting biases. We make two remarks. First, the biases are generally larger than those obtained without leakage correction. An exception is the amplitude correlation between generator 3 and 5 in the case $k=5$ and for noisy data, which is severely (positively) biased if leakage correction is omitted. After correction, the amplitude correlation essentially vanishes, which happens to agree with the almost zero true correlation between these generators. Second, the biases are predominantly negative i.e. amplitude correlations are underestimated. Negative bias of orthogonalized amplitude correlations is also visible in resting-state MEG data, where orthogonalization yields reduced correlation values [33, 48]. Our simulations hence show that although symmetric orthogonalization enables to extract the topology of functional networks, which has been shown in empirical as well as simulation studies of resting-state MEG [33, 10, 48], it generally does not allow for accurate estimates of the magnitude of amplitude correlations.

\subsection{Reconstruction of oscillatory phase-locking values}

Amplitude correlations cannot be used to infer lagged interactions between oscillatory time-series, because instantaneous amplitudes fluctuate on time-scales that are longer than the oscillation periods. In our data, for example, the oscillations have a peak-frequency of about $16 \mathrm{~Hz}$, which corresponds to a period of $62.5 \mathrm{~ms}$ (see Section 2.1). To assess if lagged interactions can be reconstructed from resting-state ECoG recordings, we repeated the simulations using phase-locking factors, instead of amplitude correlations. We only consider the case in which the number of generators $k$ is set to the true number of generators $(k=5)$, because the cases $k=4$ and $k=6$ essentially yield the same results as for amplitude correlations (see Section 3.2). We also omit the $20 \mathrm{~dB}$ case, as the reconstructions are almost identical to those obtained in the $30 \mathrm{~dB}$ case.

Figure 5A and B show the true (black) and reconstructed (red) phase-locking factors between all generator-pairs for $30 \mathrm{~dB}$ and $10 \mathrm{~dB}$ data, respectively. The phase-locking factors have been restricted to the first quadrant by taking the absolute values of their real and imaginary parts. This is done because the signs of the basis vectors in which the leadfields are expressed, are arbitrary and therefore do not allow the signs of the source time-courses to be determined. The phase-locking factors can hence be determined only modulo 180 degrees, unless the local orientations of the cortical surface are known. The figure shows that the phase-locking factors are accurately reconstructed for $30 \mathrm{~dB}$ data, while for $10 \mathrm{~dB}$ data, the reconstructions are less accurate, at least for some pairs of generators. We make several remarks about the $10 \mathrm{~dB}$ case.

First, phase-locking magnitudes i.e. interaction strengths, can be under- as well as overestimated. Second, the relative phase-differences, that is, the angles of the phase-locking factors with the positive $x$-axis, are underestimated for most pairs of generators. This effect is referred to as phase-contraction and refers to a reduction in relative phases due to volume-conduction [31]. Third, in contrast to amplitude correlations, inaccuracies in the reconstruction of phase-locking factors are not limited to a small subset of generators, but affect most pairs of generators. This reflects the fact that phases are more susceptible to volume-conduction than amplitudes [31]. Fourth, although the relative phase-differences are generally underestimated, for some generator-pairs they are overestimated. This is the case, for 
example, for generators 4 and 5: while these generators are coupled instantaneously i.e. with phasedifference zero, their reconstructed phase-locking value has non-vanishing imaginary part. Although imaginary phase-locking or coherence can never arise from a pair of instantaneously coupled generators, which has motivated the use of lagged interaction measures [46, 61], this is only true in the absence of lagged generators [13]. In the presence of lagged generators, higher-order volume-conduction effects come into play and it might happen that instantaneously coupled time-courses appear to be coupled with non-zero lag. Our simulations thus corroborate the observation made in [13] that one should be careful when interpreting the results of lagged interaction measures.

\subsection{Resting-state sensorimotor network in macaque neocortex}

In this section we apply the reconstruction pipeline to beta oscillations in the sensorimotor cortex of a single macaque monkey recorded with ECoG on two five-minute resting-state sessions (see Section 2.1). The first step in the pipeline is choosing the number of generators $k$. Figure $6 \mathrm{~A}$ shows the fraction of explained variance of the sensor-data as a function of the number of eigenvectors for session 1 (blue) and session 2 (red). The curves have no clear cut-off to separate true from noise-projected generators, although the true number of components probably does not exceed 4 . To break down the amplitude fluctuations into their smallest building blocks, we set $k=4$. Using this value, we applied spatial ICA to the session-concatenated band-limited amplitude fluctuations. To be conservative, we kept only those components that were reproducible across the two recording sessions. To identify these, we applied spatial ICA to both sessions separately, and computed the inter-session Pearson correlation between their spatial profiles. A generator was considered reproducible if its inter-session correlation exceeded 0.8. This yielded 3 generators.

The top row of Figure $6 \mathrm{~B}$ shows the amplitude profiles of the reproducible generators. Generators 1 and 3 are located in the primary motor cortex on the middle and lateral sections of the pre-central gyrus, respectively, with generator 1 extending into the frontal cortex. Generator 2 is located in the primary somatosensory cortex and covers a large part of the pre-central gyrus. Taken together, these generators hence cover large parts of the primary somatosensory and motor cortex, in line with the distributed nature of beta oscillations in macaque cortex [5]. Note that the reconstructed generators are spatially extended, in particular the somatosensory generator. Since this could be a consequence of the noise-regularization term in the inverse operator, we compared the generator's spatial profiles with the point-spread functions of the inverse operator at the generator locations (Figure 6B, bottom row). The spatial extend of the primary motor generators is comparable with that of the corresponding point-spread functions, and therefore they can indeed be explained by point-sources. The spatial extend of the somatosensory generator, however, is much larger than that of its point-spread function and, as such, cannot be explained by a point-source.

Figure $6 \mathrm{C}$ shows the band-limited amplitude correlations between all pairs of generators for both sessions. Although the exact values differ between sessions, in both sessions, the amplitude fluctuations of all three generators appear to be correlated. To assess significance, we generated 99 phase-randomized surrogate copies of the generator time-series and calculated their band-limited amplitude correlations (see Section 2.5 for details). For each pair of generators, if the observed amplitude correlation exceeds all surrogate values, the amplitude correlation is significant with $p<0.01$. All amplitude correlations for both sessions were significant with $p<0.01$. Figure $6 \mathrm{D}$ shows the phase-locking values between all pairs of generators for both sessions. They are reasonably reproducible across sessions, except for generator-pair $(2,3)$, which has a smaller phase-angle in session 2 than in session 1 . To assess significance, we used the same approach as above. All magnitudes for both sessions were significant with $p<0.01$. Note that the imaginary parts of the phase-locking values appear to be non-zero for every pair of generators. To assess for significant imaginary phase-locking, we used the same approach as above, but replacing the phase-randomized surrogate copies by symmetrized surrogate copies. This kind of randomization destroys all imaginary phase-locking, while retaining instantaneous (i.e. real- 
valued) phase-locking (see Section 2.5 for details). For session 1, imaginary phase-locking between generators 2 and 3 was significant $(p<0.01)$ and for session 2 , the imaginary phase-locking between all pairs of generators was significant $(p<0.01)$. When comparing the amplitude correlations with the phase-locking magnitudes, we see that they are both larger in session 2 than in session 1 . We suspect that this is due to the stronger beta oscillations in session 2 (see Figure 1A).

\section{Discussion}

In this study we described a method for reconstructing source-space RSNs from sensor-level ECoG data. Our approach differs from previous work in that, instead of reconstructing functional connectivity between all pairs of source-locations, functional connectivity analysis is confined to pairs of delineated generators. We considered two kinds of functional connectivity: amplitude correlations and oscillatory phase-locking. While amplitude correlations are widely used in scalp EEG and MEG studies $[12,60,40]$ and resemble the correlation patterns obtained from resting-state functional MRI $[44,34]$, amplitude signals fluctuate on time-scales that are at least one order of magnitude larger than the period of the carrier oscillations [33]. As such, they cannot be used to study oscillatory synchronization between disparate cortical oscillations, which has been hypothesized to play a fundamental role in the routing of information [19, 20]. Besides amplitude correlations, we therefore also assessed the feasibility of reconstructing source-space oscillatory phase-locking from sensor-level ECoG data, as this makes full use of the high temporal resolution of the data. Using a simulated network of cortical generators, we showed that amplitude correlations and oscillatory phase-locking can both be accurately reconstructed from sensor-level ECoG data, although oscillatory phase-locking is more challenging to reconstruct and requires a higher signal-to-noise ratio. We applied the methodology to two resting-state ECoG recording sessions of a single macaque monkey displaying strong sensorimotor beta $(\approx 16 \mathrm{~Hz})$ oscillations and found these to constitute a reproducible set of significantly coordinated generators covering primary and higher-order motor regions as well as primary somatosensory regions. This characterization of the sensorimotor network hence reveals a level of spatiotemporal detail that is unattained with MEG/EEG, which commonly characterize this network by a single generator in each hemisphere $[4,33]$. Our study thus shows that ECoG recordings allow for a more detailed characterization of the organization of resting-state cortical oscillations than can be obtained with scalp EEG and MEG, albeit at the expense of spatial coverage.

Our simulations show that source-space amplitude correlations can be accurately reconstructed from sensor-level ECoG data, even if the signal-to-noise ratio is low. Reconstruction of oscillatory phaselocking is possible as well, but requires a higher signal-to-noise ratio. At first sight, one might suspect that oscillatory phase-locking could be estimated more accurately for noisy data if the dipole orientations are assumed to be known when projecting the data to source-space. This is not the case, however, as the reconstructed phase-locking factors remain practically unchanged when the dipole orientations are assumed to be known (results not shown). Rather, instantaneous phases are more susceptible to volume-conduction than amplitudes [31] and hence are more difficult to reconstruct. We have not explicitly evaluated the performance of lagged functional connectivity indices such as the phase-lag index [61] and imaginary coherency [46], which are designed to measure functional connectivity in the presence of volume-conduction. We instead focused on the more fundamental question of how volumeconduction distorts the timing between oscillatory time-series, as characterized by the complex-valued phase-locking factor as this provides a reference for interpreting the results obtained from such interaction measures. Although relative phases are generally underestimated [31], we found that second-order volume-conduction effects [13] can lead to overestimation as well. For instance, it can happen that generators that are instantaneously coupled i.e. with zero lag, seem coupled with a non-zero lag (see Section 3.3). This unfortunate situation complicates the interpretation of lagged functional connectivity measures that only take into account first-order volume-conduction effects [46,61] and accurate reconstruction of synchronization dynamics requires the development of higher-order functional con- 
nectivity measures [13].

Our study provides evidence that the sensorimotor RSN comprises at least three cortical generators that cover primary and higher-order motor and primary somatosensory regions. The generation of beta oscillations within the primary motor cortex is well-established and can also be observed noninvasively in humans using scalp EEG and MEG [28, 36, 56, 7]. The existence of beta generators in frontal (pre-motor) regions is also well-established from invasive (LFP) recordings in macaque monkeys [58], but is not commonly reported in human EEG and MEG studies and the same is true for post-central regions [5]. ECoG source-modeling hence yields a more detailed characterization of the functional organization of sensorimotor oscillation than scalp EEG and MEG and resemble the findings reported in intra-cortical LFP studies. Our study also provides evidence for functional interactions between motor and somatosensory generators, in line with intra-cortical LFP recordings from multiple intra-cortical electrodes located in pre- and post-central regions [58, 5]. In particular, in [5], directed oscillatory coupling was reported between beta oscillations within the primary and pre-motor cortices and somatosensory cortex, including the intra-parietal sulcus. In our study we did observe relatively large latencies between the beta oscillations originating from pre- and post-central regions, which is at least consistent with the directional coupling from somatosensory to motor cortex observed in [5].

To project the sensor-level ECoG data to the cortical surface, we have used a smoothed version of the minimum norm estimate (MNE) [52]. We have also experimented with normalized inverse methods such as dynamic statistical parametric mapping (dSPM), standardized low-resolution electromagnetic tomography (sLORETA), and the weighted minimum-norm estimate (wMNE) [23], which all yielded less accurate results. The reason for this is that ECoG leadfields have a much broader range (as measured by their Euclidean norms) than scalp EEG and MEG leadfields, which is due to the limited coverage of the electrode grid. As a consequence of the normalization, the source reconstructions are typically biased towards locations that are far away from the grid. These findings are in line with another ECoG simulation study, which found MNE to outperform both sLORETA and WMNE [8]. In another study, however, WMNE was found to yield reasonable reconstructions [66], although no comparison with other methods was made. In [15], the Multiple Signal Classification (MUSIC) algorithm was found to perform well on ECoG data, although it was not directly compared to other methods. Another option are adaptive spatial filters (beamformers) [23]. Unlike in the case of scalp EEG and MEG, however, in which the equivalent dipole approximation might be reasonable, the close proximity of the ECoG electrodes to the cortex requires simulation of spatially-extended generators, at least for resting-state data. The ensuing coherence between nearby oscillations might prevent successful application of beamformers to ECoG data, as they violate the assumption of uncorrelated sources that underlies this class of methods. It thus seems that, as for intra-cortical LFP recordings [32], inverse methods that have been designed for scalp EEG and MEG data, need to be adjusted to be applicable to ECoG data.

In order to obtain a realistic idea of the performance and applicability of the proposed methodology, several limitations need to be mentioned. First, a question that we did not address in our study is how to select an appropriate number of components in the ICA decomposition. Although in the simulated data, the eigenspectra of the sensor covariance matrices had a clear elbow-shape -which can be used to select the appropriate number of components - this was not the case for the eigenspectra of the empirical data. We note that this issue not only plays a role in our study, but is inherent to matrix factorization methods in general. We have therefore chosen a pragmatic approach by requiring the components to be present in each of the two recording sessions. This approach can be viewed as a variant of split-half reliability for one data-set [62]. Second, our simulated functional networks contained five generators and it is unclear if the methodology is successful when more generators are active. We do note, however, that networks of this size are common in methodological studies on MEG/EEG source-space functional connectivity reconstruction (see, for example, [13, 10, 65]). Another potential limitation of the proposed methodology is that weak generators might be missed in 
the generator extraction step, although we did not observed this in our simulations. In other words, reducing the network size by pre-selecting the network nodes (i.e. generators) might come at the cost of missing weak nodes. An interesting question for future research, therefore, is to directly compare our method with seed-based functional connectivity methods that take into account all source locations [48]. Fourth, although we have conducted an exploratory analysis of the internal structure of one of the generators, it will be important in future research to systematically assess the accuracy of such an analysis and to see if it is applicable to scalp EEG and MEG data. Besides these methodological limitations, an obvious limitation of our study is the use of data from a single monkey. Although we have shown that the functional organization of cortical sensorimotor oscillations can be reproduced in a separate recording session, the level of inter-subject variability remains unknown. Despite these limitations, we believe that our study provides an important step in the development of ECoG sourceimaging and network reconstruction methods. Further development of this emerging field will allow

monitoring cortical function with higher spatial resolution that scalp EEG and MEG and with wider coverage than intra-cortical LFP recordings.

\section{Acknowledgments}

RH and GD were funded by the European Research Council (Advanced Grant DYSTRUCTURENo.295129), the Spanish Research Project PSI2013-42091-P, the CONSOLIDER- INGENIO2010 Program CSD200700012, and the FP7-ICT Brainscales(269921). DM was funded by the Wellcome Trust (grant 101253/Z/13/Z), KU Leuven Research Office (grant C16/15/070), Research Foundation Flanders (FWO) (grants G0F76.16N and G0936.16N). PF acknowledges grant support by DFG (SPP 1665, FOR 1847, FR2557/5-1-CORNET, FR 2557/6-1-NeuroTMR), EU (HEALTHF22008200728-BrainSynch, FP7-604102-HBP, FP7-600730Magnetrodes), a European Young Investigator Award, NIH (1U54MH091657-WU-Minn-Consortium$\mathrm{HCP})$, and LOEWE (NeFF). The authors declare no competing financial interests. 


\section{Figure legends}

Figure 1: Electrocorticographic recordings. A. Electrode-averaged power spectra for each of the two five-minute recordings sessions (session 1 (blue) and session 2 (red)). Note the presence of strong beta oscillations with a peak-frequency of about $16 \mathrm{~Hz}$. B. Ten-second traces of the 20 most anterior electrodes.

Figure 2: Simulated data. A. Amplitude profiles of the five simulated cortical generators scattered throughout the somatosensory, motor, and premotor cortices. The black dots correspond to the locations of the coregistered ECoG electrodes. B. Ten-second traces of simulated ECoG data of the 20 most anterior electrodes are shown.

Figure 3: Delineation of cortical generators. A. Pearson correlations between the spatial profiles of the true and reconstructed generators for all three noise-levels $(10,20$, and $30 \mathrm{~dB})$ for the case that the number of generators was set to $k=5$. B. Spatial profile of generator 3 and its reconstruction for all three noise-levels. C. Pearson correlations between the spatial profiles of the true and reconstructed generators for all three noise-levels for the case that the number of generators was set too high $(k=6)$. D. Spatial profile of the spurious generator $(k=6)$. It comprises generator 5 and projected sensornoise. E. Pearson correlations between spatial profiles of the true and reconstructed generators for all three noise-levels for the case that the number of generators was set too low $(k=4)$. All results were obtained by averaging over ten realizations of simulated data.

Figure 4: Reconstruction of band-limited amplitude correlations. A. Reconstructed amplitude correlations for the case $k=5$. Top row: Reconstructed amplitude correlations between all pairs of generators for all three noise-levels $(10,20$, and $30 \mathrm{~dB})$. Middle row: Corresponding biases. Bottom row: Corresponding biases after leakage correction through symmetric orthogonalization. B. Same format as A. but with the number of generators set to $k=6$. The panels show the correlations between the five true generators and the five best-matching reconstructed generators. C. Same format as A. but with the number of generators set to $k=4$. The panels show the correlations between the four reconstructed generators and the four best-matching true generators.

Figure 5: Reconstruction of oscillatory phase-locking values. A. True (black) and reconstructed (red) complex-valued phase-locking values between all pairs of generators for $10 \mathrm{~dB}$ simulated data. B. True (black) and reconstructed (red) complex-valued phase-locking values between all pairs of generators for $30 \mathrm{~dB}$ simulated data. In panels $\mathrm{A}$ and $\mathrm{B}$, the positive horizontal (i.e. real-valued) axis corresponds to a phase of zero degrees. All values displayed in panels A. and B. were obtained by averaging over ten realizations of simulated data and the number of generators was set to the true number of generators $(k=5)$.

Figure 6: Generators of resting-state sensorimotor beta oscillations in macaque neocortex. A. Fraction of explained variance of the sensor-data as a function of the number of eigenvectors for session 1 (blue) and session 2 (red). B. Top row: Generators that were present in both recording sessions, obtained by setting $k=4$ in the ICA decomposition. Bottom row: Point-spread functions at the corresponding locations. C. Band-limited amplitude correlations between all pairs of generators for session 1 (left panel) and session 2 (right panel). D. Complex phase-locking values between the oscillatory timecourses of all pairs of generators for session 1 (black lines) and session 2 (red lines). All results were obtained from a single monkey. 


\section{References}

[1] Y. Aoki, R. Ishii, R.D. Pascual-Marqui, L. Canuet, S. Ikeda, M. Hata, K. Imajo, H. Matsuzaki, T. Musha, T. Asada, M. Iwase, and M. Takeda. Detection of EEG-resting state independent networks by eLORETA-ICA method. Front. Hum. Neurosci., 9(February):31, 2015.

[2] A. Bahramisharif, M. A. J. van Gerven, E. J. Aarnoutse, M. R. Mercier, T. H. Schwartz, J. J. Foxe, N. F. Ramsey, and O. Jensen. Propagating neocortical gamma bursts are coordinated by traveling alpha waves. J. Neurosci., 33(48):18849-54, nov 2013.

[3] Steven L Bressler and Craig G Richter. ScienceDirect Interareal oscillatory synchronization in top-down neocortical processing. Curr. Biol., 31:62-66, 2015.

[4] M.J. Brookes, M. Woolrich, H. Luckhoo, D. Price, J.R. Hale, M.C. Stephenson, G.R. Barnes, S.M. Smith, and P.G.q Morris. Investigating the electrophysiological basis of resting state networks using magnetoencephalography. Proc. Natl. Acad. Sci. U. S. A., 108(40):16783-8, oct 2011.

[5] A. Brovelli, M. Ding, A. Ledberg, Y. Chen, R. Nakamura, and S.L. Bressler. Beta oscillations in a large-scale sensorimotor cortical network: directional influences revealed by Granger causality. Proc. Natl. Acad. Sci. U. S. A., 101(26):9849-54, 2004.

[6] F. Brunet, A. Bartoli, and L. Uda. L-tangent norm: A low computational cost criterion for choosing regularization weights and its use for range surface reconstruction. Proc. Fourth Int. Symp. 3D Date Process. Vis. Transm., 2008.

[7] D.O. Cheyne. MEG studies of sensorimotor rhythms: A review. Exp. Neurol., 245:27-39, 2013.

[8] J. H. Cho, S. B. Hong, Y. J. Jung, H. C. Kang, H. D. Kim, M. Suh, K. Y. Jung, C.H. Im, and Chang-Hwan Im. Evaluation of algorithms for intracranial EEG (iEEG) source imaging of extended sources: feasibility of using iEEG source imaging for localizing epileptogenic zones in secondary generalized epilepsy. Brain Topogr., 24(2):91-104, jun 2011.

[9] J. H. Cho, H. C. Kang, Y.-J. Jung, J.-Y. Kim, H. D. Kim, D. S. Yoon, Y. H. Lee, and C. H. Im. Localization of epileptogenic zones in Lennox-Gastaut syndrome using frequency domain source imaging of intracranial electroencephalography: a preliminary investigation. Physiol. Meas., 34(2):247-63, feb 2013.

[10] G. L. Colclough, M. J. Brookes, S. M. Smith, and M. W. Woolrich. A symmetric multivariate leakage correction for MEG connectomes. Neuroimage, 117:439-448, 2015.

[11] J. S. Damoiseaux, S. A. R. B. Rombouts, F. Barkhof, P. Scheltens, C. J. Stam, S. M. Smith, and C. F. Beckmann. Consistent resting-state networks across healthy subjects. Proc. Natl. Acad. Sci., 103(37):13848-13853, 2006.

[12] F. de Pasquale, S. Della Penna, A.Z. Snyder, C. Lewis, D. Mantini, L. Marzetti, Paolo Belardinelli, Luca Ciancetta, Vittorio Pizzella, Gian Luca Romani, and Maurizio Corbetta. Temporal dynamics of spontaneous MEG activity in brain networks. Proc. Natl. Acad. Sci. U. S. A., 107(13):60406045,2010

[13] M. Drakesmith, W. El-Deredy, and S. Welbourne. Reconstructing coherent networks from electroencephalography and magnetoencephalography with reduced contamination from volume conduction or magnetic field spread. PLoS One, 8(12), 2013.

[14] M. Dumpelmann, T. Ball, and A. Schulze-bonhage. sLORETA allows reliable distributed source reconstruction based on subdural strip and grid Recordings. Human, 33:1172-1188, 2012. 
[15] M. Dümpelmann, J. Fell, J. Wellmer, H. Urbach, and C. E. Elger. 3D source localization derived from subdural strip and grid electrodes: a simulation study. Clin. Neurophysiol., 120(6):1061-9, jun 2009.

[16] Gaute T Einevoll, Christoph Kayser, Nikos K Logothetis, and Stefano Panzeri. Modelling and analysis of local field potentials for studying the function of cortical circuits. Nat. Rev. Neurosci., 14(11):770-85, nov 2013.

[17] Q. Fang and D.A. Boas. Tetrahedral mesh generation from volumetric binary and gray-scale images. (Isbi), 2009.

[18] M. D. Fox, D. Zhang, A. Z. Snyder, and M. E. Raichle. The Global Signal and Observed Anticorrelated Resting State Brain Networks. J. Neurophysiol., 101(6):3270-3283, 2009.

[19] P. Fries. A mechanism for cognitive dynamics: neuronal communication through neuronal coherence. Trends Cogn. Sci., 9(10):474-80, oct 2005.

[20] P. Fries. Rhythms for Cognition: Communication through Coherence. Neuron, 88(1):220-235, 2015.

[21] M. Fuchs, M. Wagner, and J. Kastner. Development of volume conductor and source models to localize epileptic Foci. J. Clin. Neurophysiol., 24(2):101-119, 2007.

[22] A. Gramfort, T. Papadopoulo, E. Olivi, and M. Clerc. OpenMEEG : opensource software for quasistatic bioelectromagnetics. pages 1-20, 2010.

[23] R. Grech, T. Cassar, J. Muscat, K. P. Camilleri, S. G. Fabri, M. Zervakis, P. Xanthopoulos, V. Sakkalis, and B.k Vanrumste. Review on solving the inverse problem in EEG source analysis. J. Neuroeng. Rehabil., 5(25), jan 2008.

[24] D.A. Gusnard and M.E. Raichle. Searching for a baseline: Functional imaging and the resting human brain. Nat. Rev. Neurosci., 2(10):685-694, 2001.

[25] C.D. Hacker, A.Z. Snyder, M. Pahwa, M. Corbetta, and E.C. Leuthardt. Frequency-specific electrophysiologic correlates of resting-state fMRI networks. Neuroimage, 149(January):446-457, 2017.

[26] M. Hamalainen, R. Hari, J. Ilmoniemi, J. Knuutila, and O.V. Lounasmaa. Magnetoencephalography - theory, instrumentation, and applications to noninvasive studies of the working human brain. Rev. Mod. Phys., 65(2), 1993.

[27] P.C. Hansen and D.P. O'Leary. The use of the L-curve in the regularization of discrete ill-posed problems. SIAM J. Sci. Comput., 14(6):1487-1503, 1993.

[28] R. Hari and R. Salmelin. Human cortical oscillation: a neuromagnetic view through the skull. Trends Neurosci., 20(1):44-49, 1997.

[29] B.J. He, A.Z. Snyder, J.M. Zempel, M.D. Smyth, and M.E. Raichle. Electrophysiological correlates of the brain's intrinsic large-scale functional architecture. Proc Natl Acad Sci U S A, 105(41):16039-16044, 2008.

[30] A. Hillebrand, G.R. Barnes, J.L. Bosboom, H.W. Berendse, and C.J. Stam. Frequency-dependent functional connectivity within resting-state networks: An atlas-based MEG beamformer solution. Neuroimage, 59(4):3909-3921, 2012.

[31] R. Hindriks, X.D. Arsiwalla, T. Panagiotaropoulos, M. Besserve, P.F.M.J. Verschure, N.K. Logothetis, and G. Deco. Discrepancies between Multi-Electrode LFP and CSD Phase-Patterns: A Forward Modeling Study. Front. Neural Circuits, 10(July):51, 2016. 
[32] R. Hindriks, J. Schmiedt, X.D. Arsiwalla, A. Peter, P.F.M.J. Verschure, P. Fries, M.C. Schmid, and G. Deco. Linear distributed source modeling of local field potentials recorded with intracortical electrode arrays. PLoS One, 12(12), 2017.

[33] J.F. Hipp, D.J. Hawellek, M. Corbetta, M. Siegel, and A.K. Engel. Large-scale cortical correlation structure of spontaneous oscillatory activity. Nat. Neurosci., 15(6):884-90, jun 2012.

[34] J.F. Hipp and M. Siegel. BOLD fMRI correlation reflects frequency-specific neuronal correlation. Curr. Biol., 25(10):1368-1374, 2015.

[35] R. Matthew Hutchison, L. Stan Leung, Seyed M. Mirsattari, Joseph S. Gati, Ravi S. Menon, and Stefan Everling. Resting-state networks in the macaque at 7T. Neuroimage, 56(3):1546-1555, 2011.

[36] O Jensen, P Goel, N Kopell, M Pohja, R Hari, and B Ermentrout. On the human sensorimotorcortex beta rhythm: sources and modeling. Neuroimage, 26(2):347-55, jun 2005.

[37] Y. Jonmohamadi and R.D. Jones. Source-space ICA for MEG source imaging. J Neural Eng, 13(1):16005, 2016.

[38] Y. Kajikawa and C. E. Schroeder. How local is the local field potential? Neuron, 72(5):847-58, $\operatorname{dec} 2011$.

[39] J. S. Kim, C. H. Im, Y. J. Jung, E. Y. Kim, S. K. Lee, and C. K. Chung. Localization and propagation analysis of ictal source rhythm by electrocorticography. Neuroimage, 52(4):1279-88, oct 2010 .

[40] Q. Liu, S. Farahibozorg, and C. Porcaro. Detecting large-scale networks in the human brain using high-density electroencephalography. BioRxiv, pages 1-31, 2016.

[41] L. Manola, B. H. Roelofsen, J. Holsheimer, E. Marani, and J. Geelen. Modelling motor cortex stimulation for chronic pain control: electrical potential field, activating functions and responses of simple nerve fibre models. Med. Biol. Eng. Comput., 43(3):335-343, 2005.

[42] D. Mantini, M. Corbetta, G. L. Romani, G. A. Orban, and W. Vanduffel. Evolutionarily novel functional networks in the human brain? J. Neurosci., 33(8):3259-75, feb 2013.

[43] D. Mantini, S. D. Penna, L. Marzetti, F. de Pasquale, V. Pizzella, M. Corbetta, and G. L. Romani. A Signal-Processing Pipeline for Magnetoencephalography Resting-State Networks. Brain Connect., 1(1):49-59, 2011.

[44] D. Mantini, M.G. Perrucci, C. Del Gratta, G.L. Romani, and M. Corbetta. Electrophysiological signatures of resting state networks in the human brain. Proc. Natl. Acad. Sci., 104(32):13170, 2007.

[45] D.G. Mclaren, K.J. Kosmatka, T.R. Oakes, D. Christopher, S.G. Kohama, J.A. Matochik, D.K. Ingram, and C. Sterling. A population-average MRI-based atlas collection of the rhesus macaque. Neuroimage, 45(1):52-59, 2010.

[46] G. Nolte, O. Bai, L. Wheaton, Z. Mari, S. Vorbach, and M. Hallett. Identifying true brain interaction from EEG data using the imaginary part of coherency. Clin. Neurophysiol., 115(10):2292-307, oct 2004 .

[47] Paul L. Nunez and R. Srinivasan. Electric fields of the brain., volume second edi.

[48] G.C. O’Neill, E.L. Barratt, B.A.E. Hunt, P.K. Tewarie, and M.J. Brookes. Measuring electrophysiological connectivity by power envelope correlation: a technical review on MEG methods. Phys. Med. Biol., 60(21):R271-R295, 2015. 
[49] T.F. Oostendorp and A. van Oosterom. Source parameter estimation in inhomogeneous volume conductors of arbitrary shape. IEEE Trans. Biomed. Eng., 36(3):382-391, 1989.

[50] R. Oostenveld, P. Fries, E. Maris, and J.-M. Schoffelen. FieldTrip: Open source software for advanced analysis of MEG, EEG, and invasive electrophysiological data. Comput. Intell. Neurosci., page 156869 , jan 2011.

[51] E. Pereda, R. Quian, and J. Bhattacharya. Nonlinear multivariate analysis of neurophysiological signals. Prog. Neurobiol., 77:1-37, 2005.

[52] Y. Petrov. Harmony: EEG/MEG linear inverse source reconstruction in the anatomical basis of spherical harmonics. PLoS One, 7(10):e44439, jan 2012.

[53] K.H. Pettersen, A. Devor, I. Ulbert, A.M. Dale, and G.T. Einevoll. Current-source density estimation based on inversion of electrostatic forward solution: effects of finite extent of neuronal activity and conductivity discontinuities. J. Neurosci. Methods, 154(1-2):116-33, jun 2006.

[54] D. Prichard. Generating surrogate data for time series with several simultaneously measured variables. Phys. Rev. Lett., 73(7):951-954, 1994.

[55] G. Ramantani, D. Cosandier-Rimélé, A. Schulze-Bonhage, L. Maillard, J. Zentner, and M. Dümpelmann. Source reconstruction based on subdural EEG recordings adds to the presurgical evaluation in refractory frontal lobe epilepsy. Clin. Neurophysiol., 124(3):481-91, mar 2013.

[56] P. Ritter, M. Moosmann, and A. Villringer. Rolandic alpha and beta EEG rhythms' strengths are inversely related to fMRI-BOLD signal in primary somatosensory and motor cortex. Hum. Brain Mapp., 30(4):1168-1187, 2009.

[57] B. Rubehn, C. Bosman, R. Oostenveld, P. Fries, and T. Stieglitz. A MEMS-based flexible multichannel ECoG-electrode array. J. Neural Eng., 6(3):036003, jun 2009.

[58] J.N. Sanes and J.P. Donoghue. Oscillations in local field potentials of the primate motor cortex during voluntary movement. Proc. Natl. Acad. Sci. U. S. A., 90(10):4470-4474, 1993.

[59] T. Schreiber and A. Schmitz. Surrogate time series. Phys. D Nonlinear Phenom., 142:346-382, 2000 .

[60] Marcus Siems, Anna Antonia Pape, Joerg F. Hipp, and Markus Siegel. Measuring the cortical correlation structure of spontaneous oscillatory activity with EEG and MEG. Neuroimage, 129:345-355, 2016.

[61] C.J. Stam, G. Nolte, and A. Daffertshofer. Phase lag index: assessment of functional connectivity from multi channel EEG and MEG with diminished bias from common sources. Hum. Brain Mapp., 28(11):1178-93, nov 2007.

[62] Roemer van der Meij, Joshua Jacobs, and Eric Maris. Uncovering phase-coupled oscillatory networks in electrophysiological data. Hum. Brain Mapp., 36(7):2655-2680, 2015.

[63] Roemer van der Meij, Michael Kahana, and Eric Maris. Phase-Amplitude Coupling in Human Electrocorticography Is Spatially Distributed and Phase Diverse. J. Neurosci., 32(1):111-123, 2012.

[64] D. C. Van Essen, H. A. Drury, J. Dickson, J. Harwell, D. Hanlon, and C. H. Anderson. An Integrated Software Suite for Surface-based Analyses of Cerebral Cortex. 8(5):443-459, 2001.

[65] Vincent Wens, Brice Marty, Alison Mary, Mathieu Bourguignon, Marc Op de Beeck, Serge Goldman, Patrick Van Bogaert, Philippe Peigneux, and Xavier De Ti??ge. A geometric correction scheme for spatial leakage effects in MEG/EEG seed-based functional connectivity mapping. Hum. Brain Mapp., 36(11):4604-4621, 2015. 
[66] Y. Zhang, W. Drongelen, van, M. Kohrman, and B. He. NeuroImage Three-dimensional brain current source reconstruction from intra-cranial ECoG recordings. Neuroimage, 42:683-695, 2008. 
Figure 1

Cli\&k here to download 9. Figure: figure_1.pdf B
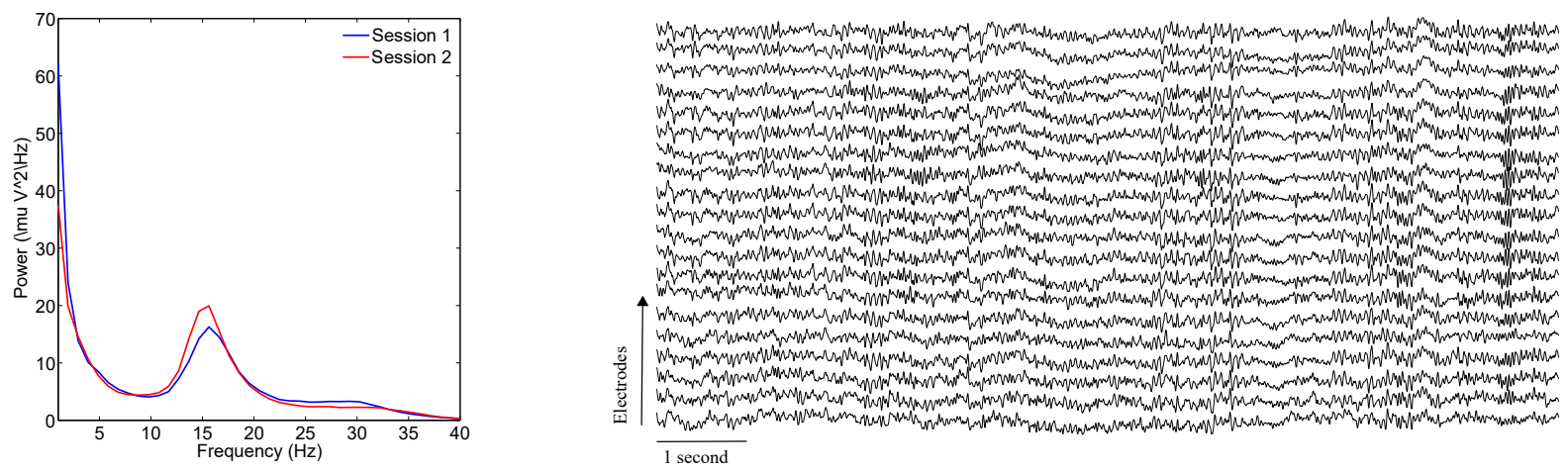
Figure 2

Click here to download 9. Figure: figure_2.pdf

A
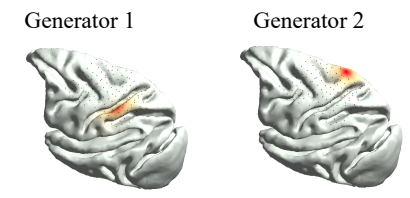

Generator 3

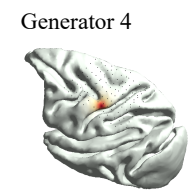

Generator 5

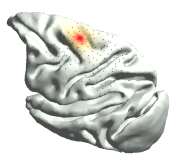

B

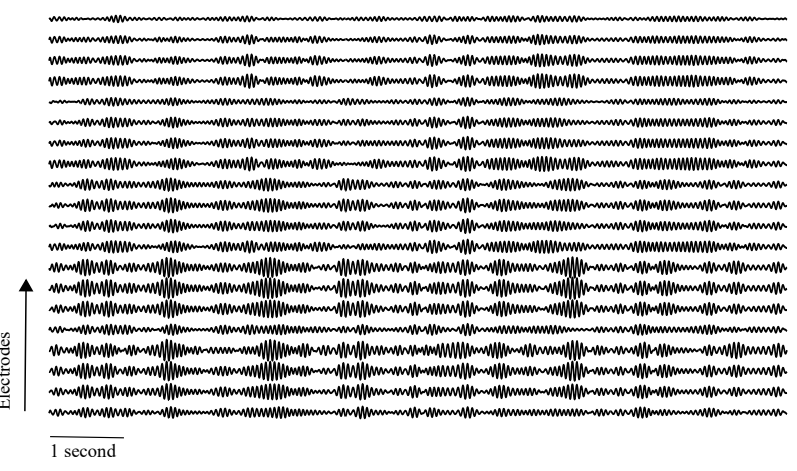


Figure 3

Click here to download 9. Figure: figure_3.pdf
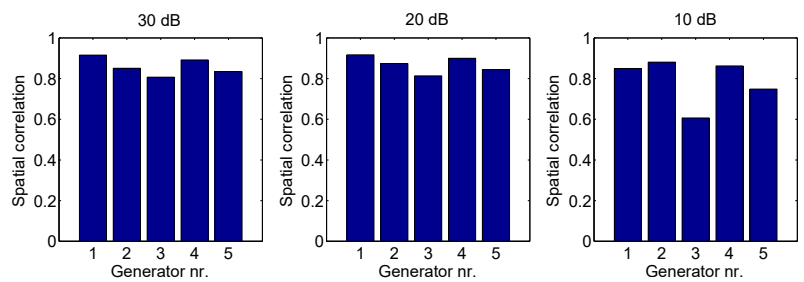

C
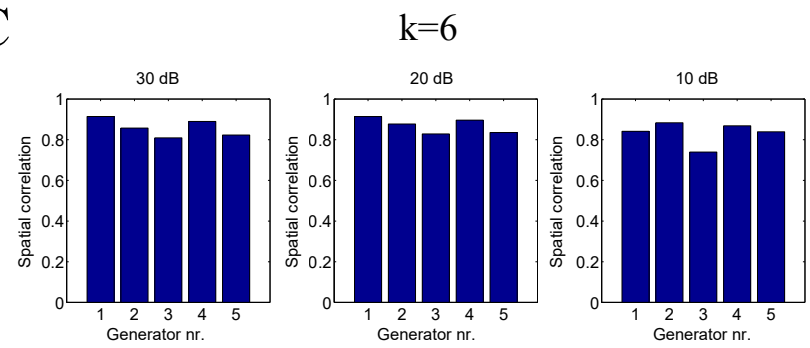

E

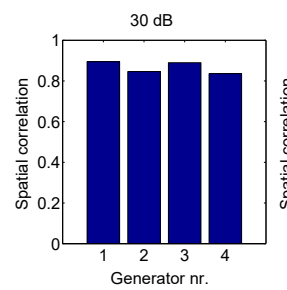

$\mathrm{k}=4$

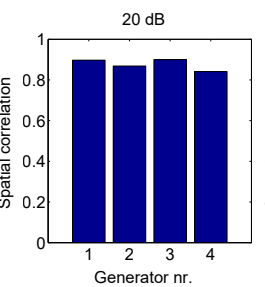

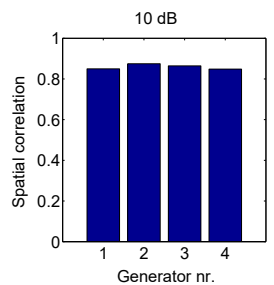

B

Reconstruction of generator 3
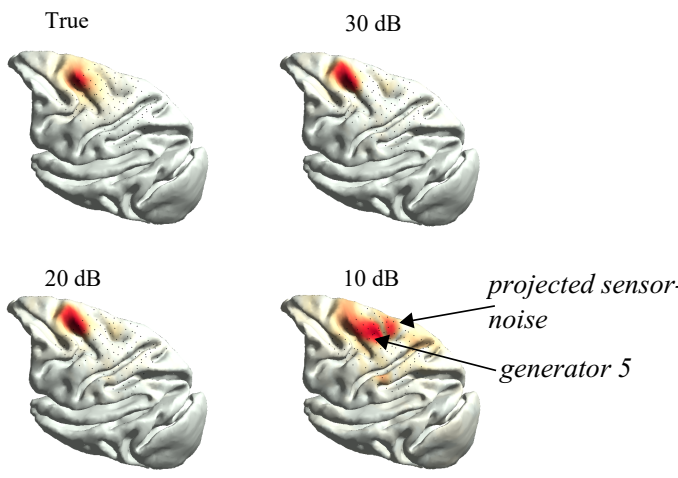

D

Spurious generator

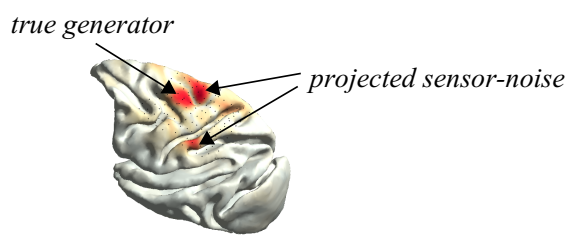


Figure 4

B

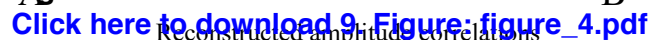

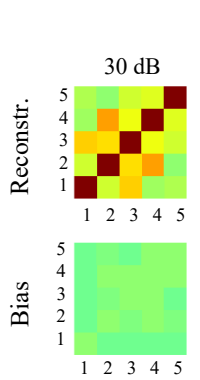

$$
(\mathrm{k}=5)
$$
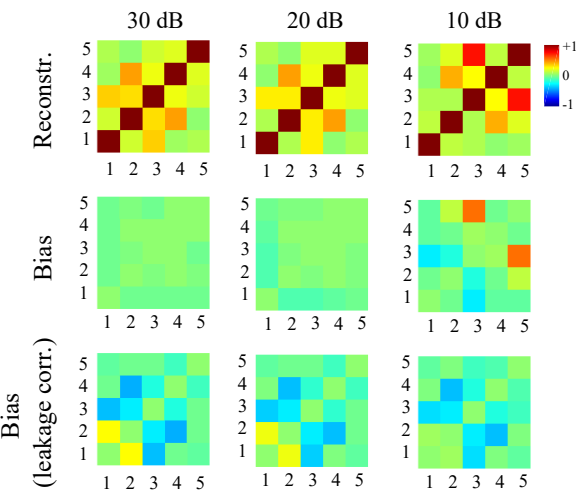

Reconstructed amplitude correlations $(\mathrm{k}=6)$
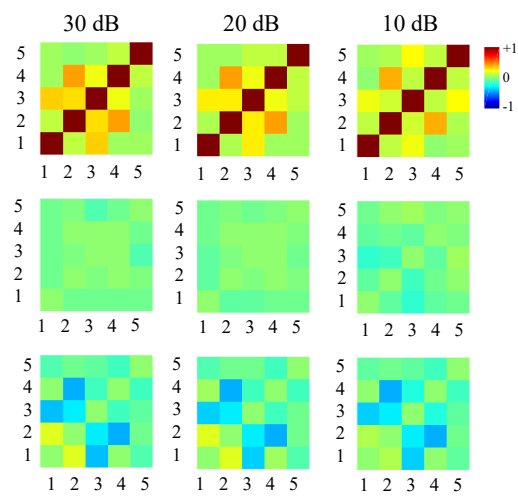

$\mathrm{C}$

Reconstructed amplitude correlations $(\mathrm{k}=4)$
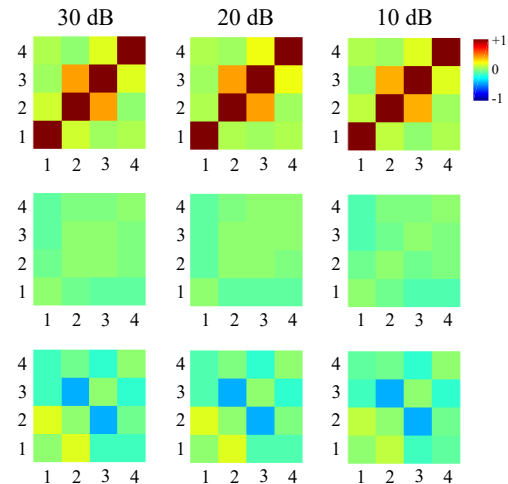
Figure 5

Click here to download 9. Figure: figure_5.pdf

Reconstruction of phase-locking values (SNR of $30 \mathrm{~dB}$ )

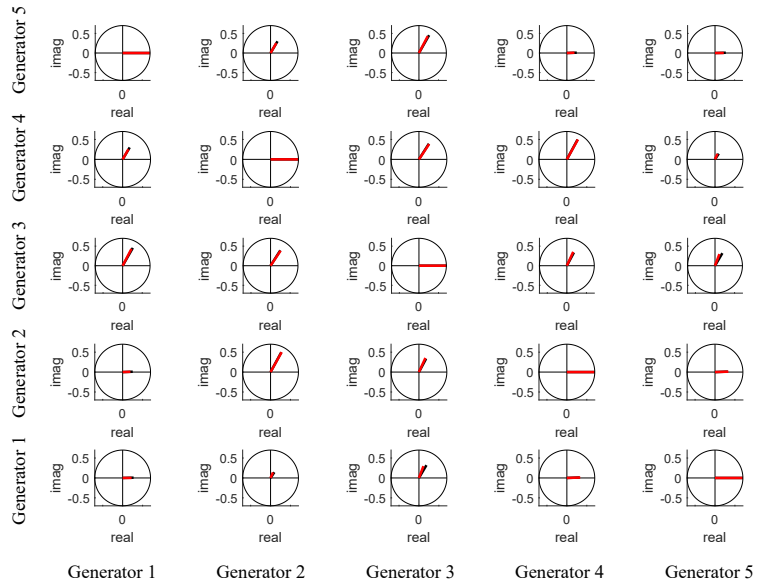

B

Reconstruction of phase-locking values (SNR of $10 \mathrm{~dB}$ )

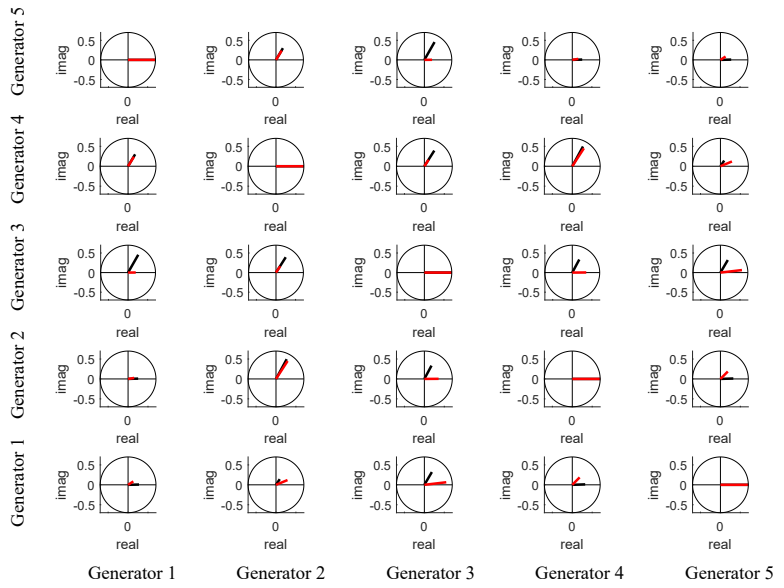

True

Reconstructed 


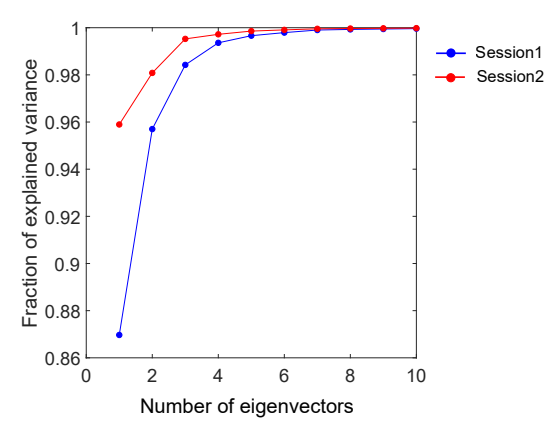

C

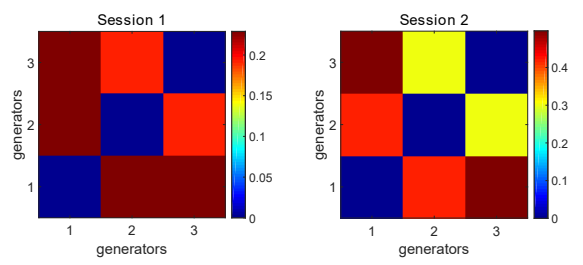

generator 1

(primary motor)

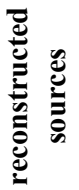

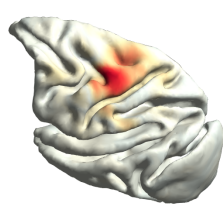

.

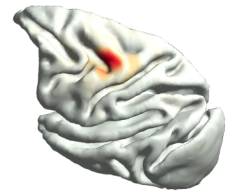

generator 2

(primary somatosensory)
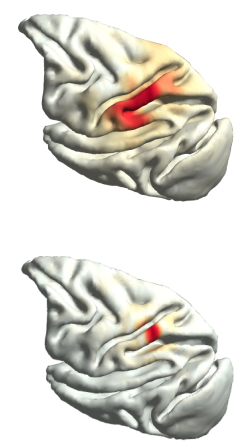

D
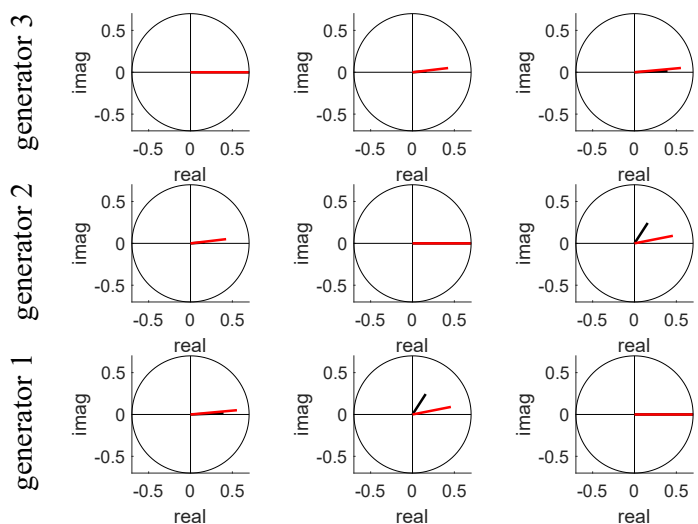

generator 1

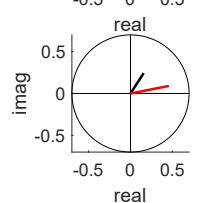

generator 2
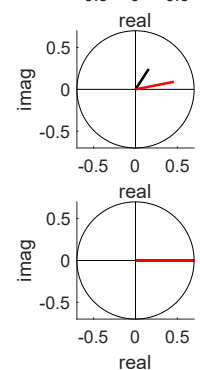

generator 3 generator 3

(primary motor)
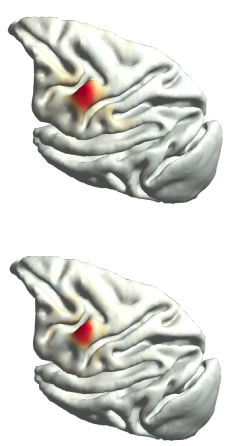